This is the final peer-reviewed accepted manuscript of:

Fleiner, T., Haussermann, P., Mellone, S., \& Zijlstra, W.

Sensor-based assessment of mobility-related behavior in dementia: Feasibility and relevance in a hospital context

In:

International Psychogeriatrics, 2016, 28(10), 1687-1694

The final published version is available online at:

https://doi.org/10.1017/S1041610216001034

Rights / License:

The terms and conditions for the reuse of this version of the manuscript are specified in the publishing policy. For all terms of use and more information see the publisher's website.

This item was downloaded from IRIS Università di Bologna (https://cris.unibo.it/)

When citing, please refer to the published version. 


\title{
Sensor-based assessment of mobility-related behavior in dementia: feasibility and relevance in a hospital context
}

Fleiner, Tim, MA, ${ }^{1,2}$; Haeussermann, Peter, MD, ${ }^{2}$; Mellone, Sabato, $\mathrm{PhD},{ }^{3} ;$ Zijlstra, Wiebren, $\mathrm{PhD}^{2}$

1. Institute of Movement and Sport Gerontology, German Sport University Cologne, Germany,

2. LVR-Klinik Cologne, Department of Geriatric Psychiatry, Germany

3. Department of Electrical, Electronic, and Information Engineering "Guglielmo Marconi", University of Bologna, Italy

\begin{abstract}
BACKGROUND

The assessment of patients' motor behavior is a key challenge in dementia care. Common geriatric assessment questionnaires or actigraphy measurements often lack methodological quality and are unsuitable to individually tailor interventions. Hence, there is a need for developing objective tools to assess patterns of motor behavior. Therefore, the feasibility of a sensor-based assessment of mobility-related behavior in patients with dementia is investigated.
\end{abstract}

\section{METHODS}

A cross-sectional investigation on three dementia care wards in a psychiatric hospital was conducted. Fortyfive patients with stages of dementia were included. Hybrid motion sensors, recording the sequence of bodypostures, were attached on the patients' lower back for 72 consecutive hours.

\section{RESULTS}

Eighty-nine percent of the assessment periods were completed. On average patients spent $10.9 \mathrm{~h} /$ day lying (45\%), 9.7h/day sedentary while sitting or standing (41\%), 1.7h/day active while sitting or standing (7\%), $1.7 \mathrm{~h} /$ day walking $(7 \%)$ and reached on average 8829 steps per day $(\mathrm{SD}=7428)$. Though overall activity levels were low, the results indicate a wide spectrum of activity patterns - ranging from almost inactive to highly active with general restlessness and wandering behavior.

\section{CONCLUSION}

The excellent adherence to the assessment protocol compared to wrist-worn actigraphy and the consistency of the sensor-derived analyses with clinical observations are pivotal findings of this study. These results show that it is possible to acquire objective data on individual motor behavior of patients suffering from dementia. This information is essential for tailoring the therapeutic management of these patients in a hospital context. 


\section{INTRODUCTION}

Dementia presents one of the most challenging healthcare issues in present and will be in future. The decline of cognitive functioning is a central part of this neurodegenerative disease. In an advanced stage of dementia, the incidence of behavioral and psychological symptoms is regarded to be more challenging for caregivers than the cognitive impairment itself. Behavioral and psychological symptoms of dementia (BPSD) include "symptoms of disturbed perception, thought content, mood, or behavior that frequently occur in patients with dementia" (Draper et al., 2015, p. 1.5). Behavioral symptoms include physical aggression, screaming, restlessness, agitation, wandering, culturally inappropriate behaviors, sexual disinhibition, hoarding, cursing and shadowing; Psychological symptoms include anxiety, depressive mood, hallucinations and delusions (Draper et al., 2015, p. 1.5). Especially in advanced stages of dementia, BPSD often lead to hospitalization or admission into long-term care nursing homes (Zuidema et al., 2007). Institutionalization is associated with a high incidence of falls due to cognitive impairment, frailty, instability or BPSD with consecutive psychopharmacological treatment (Eriksson et al., 2009). In addition to patients' mobility impairment and high risk of falling, clinicians are confronted with aberrant motor behavior symptoms, i.e. restlessness or circadian rhythm disturbances (Lyketsos et al., 2011).

There is a plethora of evidence concerning serious side effects of antipsychotic medication in the treatment of BPSD. Thus there is an urgent need for tailoring non-pharmacological treatment approaches in patients suffering from dementia (Kales et al., 2015). Tailored interventions require appropriate clinical assessment tools as well as objective data on patients' motor behavior. These data are often not available in these patients. The frequency and severity of motor behavior symptoms are usually rated on the basis of ordinal ranking scales or dichotomized estimations, and often there is no objective classification of the severity of symptoms, since the assessment is commonly based on direct observation, video-monitoring or proxy-assessment (KingKallimanis et al., 2010). According to previous studies, the use of these subjective assessment methods can lead to an over- or underestimation of aberrant motor behavior (King-Kallimanis et al., 2010), thereby limiting its clinical use (Halek and Bartholomeyczik, 2012).

Motion sensors fixed to the body can be used to objectively assess aspects of motor behavior associated with BPSD, like general restlessness, wandering behavior and circadian rhythm disturbances of patients' motor behavior (Zijlstra and Aminian, 2007; Zijlstra et al., 2011). Regarding their use in clinical care, current motion sensors are smaller and allow for unobtrusive assessments in comparison to previous devices with cable-based data traffic and an external data storage as well as power source. Therefore, these current sensor- devices may present an interesting, new approach to assess motor behavior disturbances in dementia care and clinical research. Until now, uni- and multi-axial accelerometers have most commonly been used to log patients' motor behavior in relation to time of the day. Widely used types are actigraphs (Morgenthaler et al., 2007), commonly used in sleep medicine and chronobiological research to assess circadian motor behavior. Actigraphs accumulate raw activity counts of body or limb movements (Morgenthaler et al., 2007; Van Someren, 2011). The small watch-like devices are attached on the patient's wrist to log the periodicity of limb movements. These actigraphic data do not enable detecting specific motor behavior patterns in relation to posture, acceleration or movement direction. Actigraphs can only record the presence or absence of motor activity. Additional objective methods include the use of pedometers, attached to the patients' ankle or waist, in order to log a patient's step count over several days (Algase et al., 2003; Kirste et al., 2013). The success of applying devices on the limb or waist for several days is limited in patients with dementia because these devices are often removed especially by patients suffering from advanced dementia (Camargos et al., 2013).

The application of motion sensor systems fixed on the patient's lower back may be a more successful approach if patients get used to them and the presence of the sensor remains unnoticed. Based on three-dimensional hybrid motion sensors on the lower back, patients' body posture can be recorded over several days, which allows the analysis of individual mobility patterns as an indication of the patients' motor behavior (Zijlstra and Aminian, 2007; Zijlstra et al., 2011). However, at present it is unclear whether patients suffering from dementia tolerate the attachment of sensors on the lower back for several days. Hence, our aim was to investigate a) the feasibility of using a sensor on the lower back in patients with dementia during a period of at least 72 
consecutive hours; and b) patterns of mobility-related (in)activity in these patients during their hospital stay in specialized dementia care wards.

\section{METHODS}

\section{Study Design}

Cross-sectional study as part of a clinical RCT (German Clinical Trial Register: DRKS00006740; Fleiner et al., 2015). Ethical approval was obtained by the ethics commission of the German Sport University Cologne and the ethics commission of the North-Rhine Medical Chamber.

\section{Setting and Participants}

Patients were recruited from three specialized dementia care units of a geriatric psychiatry hospital. These were admitted to this hospital because of an acute exacerbation of BPSD -

e.g. aggressive behavior, apathy, or circadian rhythm disturbances that lead to increased endangerment of themselves and others, or extremely increased caregiver burden. Inclusion criteria are as follows: primary form of dementia according to ICD-10 classification (World Health Organisation, 1997), exclusion of delirium (Inouye et al., 1990); ability to perform the Timed Up and Go-Test (TuG) (Podsiadlo and Richardson, 1991) without personal assistance

- the use of a walking aid was no exclusion criteria. Written approval for participation either by the patient or the legal guardian was required. In accordance with the ethical approval, the following consent for participation was conducted: If the cognitive impairment as measured by the Mini Mental Status Examination (MMSE) (Folstein et al., 1975) was less than 20 points, the legal guardian of the patient had to approve the participation. If a patient had reached 20 points or more and was able to explain the content and target of the project in his own words, his decision for participation was considered as accountable.

\section{Instruments}

Cognitive functioning was assessed via MMSE (Folstein et al., 1975), Demtect (Kalbe et al., 2004) and the Clock Drawing Test (CDT) (Sunderland et al., 1989). Cognitive reserve capacity was quantified according to Stern (Stern, 2002). Patients' mobility and capability to perform activities of daily living were measured via TuG, 10 meter gait speed (Ries et al., 2009) and the Bayer instrumental activities of daily living (Hindmarch et al., 1998). The patients' neuropsychiatric symptoms were measured by the Neuropsychiatric Inventory (Cummings et al., 1994) and the Cohen-Mansfield Agitation Inventory (Cohen-Mansfield and Billing, 1986). In order to monitor the patients' motor behavior, uSense sensors (FARSEEING EU-Consortium, 2015) were attached on patients' lower back with waterproof adhesive foil (Opsite FlexiFix, Smith \& Nephew Medical Ltd., Hull, Eng). This attachment allows to remove the sensor by the patient himself. The uSense sensor is a 3-D hybrid motion sensor with integrated tri-axial accelerometer, gyroscope and magnetometer. Data were sampled at $100 \mathrm{~Hz}$ and stored on an internal storage medium. Each patient was advised to wear the sensor for 72 consecutive hours. If a patient perceived the sensor- attachment as uncomfortable or painful, the nursing staff was given one additional try to motivate the patient. If the patient repeated his unwillingness to tolerate the sensor device, the nursing staff removed the sensor device. In order to assess the feasibility of the sensor attachment in these patients, occurrence of adverse events within each assessment period were logged in a clinical protocol. Furthermore, dermatological tolerance was clinically monitored.

\section{Data-analysis}

Based on the sensor data, patients' body-postures over three consecutive days were analyzed by means of a hierarchical classification approach: activity counts at intervals of $1 \mathrm{~s}$ were calculated according to Sasaki et al. (2011) and the cut-points on the intensity of physical activity were used for distinguishing between 'sedentary' and 'active' periods. In 'sedentary' time intervals the interval was labeled as 'lying' if the angle between the vertical axis of the sensor/trunk and the horizontal plane was below $15^{\circ}$ for a prolonged period of time. 
Otherwise it was defined as 'sedentary' sitting or standing. In 'active' time intervals a step detector was used for selecting walking bouts, labeled as 'gait', otherwise time intervals were labeled as 'active' sitting or standing. Due to circadian rhythm disturbances in patients' motor behavior, only complete days of measurement, from 00:00 on the first day to 24:00 on the second day have been included in the analysis. If two complete measurement days were available, averages of both recorded days were calculated and included in further analyses. The results of the sensor-recordings of each patient were checked upon consistency with the observations of the nursing stuff based upon the routine clinical protocols. The measures of central tendency were realized via means, standard deviations, minima and maxima within the descriptive statistics. The feasibility of the sensor-based assessment method and the daily activity levels were analyzed based on these descriptive statistics. All of the aforementioned analyses were carried out in MATLAB (Release 2012b, The MathWorks, Inc., Natick, MA), subsequent statistical evaluation of the data was done in MS EXCEL (Excel 2007, Microsoft, Inc., Redmond, WA).

\section{RESULTS}

A group of 45 patients (26 women and 19 men) was included in this cross-sectional investigation. Group characteristics are presented in Table 1.

\begin{tabular}{|c|c|c|c|c|c|}
\hline Characteristic & n (\%) & $\mathbf{M}$ & SD & Min & Max \\
\hline Age & 45 & 79 & 7 & 55 & 95 \\
\hline Body mass index [kg/m²] & 45 & 26.0 & 4.2 & 17.9 & 34.0 \\
\hline \multicolumn{6}{|l|}{ Diagnosis } \\
\hline Dementia in Alzheimer's disease [F00] & $13(29)$ & & & & \\
\hline Vascular dementia [F01] & $10(22)$ & & & & \\
\hline mixed type of dementia [F02 + F03] & $17(38)$ & & & & \\
\hline Dementia in Parkinson's disease [F02.3] & $4(9)$ & & & & \\
\hline Lewy-body dementia [G31.8] & $1(2)$ & & & & \\
\hline Mini Mental Status Examination & 45 & 17.9 & 5.0 & 7.0 & 27.0 \\
\hline Demtect & 28 & 4.4 & 2.5 & 0.0 & 10.0 \\
\hline Clock Drawing Test & 34 & 5.0 & 1.4 & 1.0 & 6.0 \\
\hline Neuropsychiatric Inventory & 45 & 25.3 & 11.6 & 2 & 53 \\
\hline Cohen-Mansfield Agitation Inventory & 45 & 53.0 & 12.2 & 31 & 79 \\
\hline Cognitive Reserve Capacity, years of education & 45 & 10.9 & 2.5 & 6.0 & 18.0 \\
\hline Bayer-Activities of Daily Living & 45 & 7.3 & 1.9 & 2.3 & 9.5 \\
\hline Timed Up and Go Test [s] & 45 & 15.4 & 6.8 & 7.3 & 33.6 \\
\hline 10 Meter Gait Sped [m/s] & 45 & 1.3 & 0.4 & 0.6 & 2.9 \\
\hline \multicolumn{6}{|c|}{$\mathrm{M}=$ mean; SD = Standard Deviation; Min= Minimum; Max = Maximum } \\
\hline
\end{tabular}


Table 2. Neuropsychiatric symptoms as measured by the NPI ( $n=45)$

\begin{tabular}{l|ccccc|ccccc}
\hline & \multicolumn{5}{c|}{ patients symptoms } & \multicolumn{5}{c}{ caregiver burden } \\
\hline NPI Domains & $\mathbf{n}(\%)$ & $\mathbf{M}$ & SD & Min & Max & $\mathbf{n}(\%)$ & $\mathbf{M}$ & SD & Min & Max \\
\hline Delusions & $19(42)$ & 5.4 & 3.1 & 0 & 12 & $18(40)$ & 2.3 & 0.8 & 1 & 3 \\
Hallucinations & $10(22)$ & 5.3 & 3.5 & 0 & 12 & $9(20)$ & 2.4 & 0.9 & 1 & 4 \\
Agitation/Aggression & $31(69)$ & 5.8 & 2.8 & 2 & 12 & $31(69)$ & 2.5 & 0.8 & 1 & 4 \\
Depression/Dysphoria & $31(69)$ & 4.5 & 2.9 & 0 & 12 & $30(67)$ & 2.1 & 0.8 & 1 & 4 \\
Anxiety & $24(53)$ & 5.3 & 2.9 & 1 & 12 & $24(53)$ & 2.3 & 0.7 & 1 & 3 \\
Elation/Euphoria & $4(7)$ & 3.8 & 3.3 & 0 & 8 & $3(7)$ & 2.3 & 0.6 & 2 & 3 \\
Apathy/Indifference & $26(58)$ & 4.6 & 2.6 & 0 & 12 & $26(58)$ & 2.1 & 1.0 & 0 & 4 \\
Disinhibition & $6(13)$ & 2.5 & 2.1 & 0 & 6 & $5(11)$ & 1.6 & 1.1 & 0 & 3 \\
Irritability/Lability & $30(67)$ & 5.1 & 2.7 & 0 & 12 & $30(67)$ & 2.2 & 0.8 & 0 & 3 \\
Aberrant motor behavior & $22(49)$ & 5.7 & 3.0 & 3 & 12 & $22(49)$ & 1.9 & 0.9 & 0 & 3 \\
Sleep and night-time & $11(24)$ & 6.4 & 2.4 & 3 & 9 & $11(24)$ & 2.9 & 0.8 & 1 & 4 \\
behavior disorders & $4(9)$ & 8.5 & 5.2 & 1 & 12 & $4(9)$ & 2.8 & 0.5 & 2 & 3 \\
\hline Appetite/Eating changes & 4.4 &
\end{tabular}

patients symptoms: frequency*severity scores (0-12; 0 meaning "absent"); caregiver burden (0-5; 0 meaning "not at all"); $M=$ mean; SD = Standard Deviation; Min= Minimum; Max = Maximum

The basic screening tool for dementia in our study was the MMSE. All included patients, managed to perform the MMSE. Cognitive performance of all patients ranged from mild to severe cognitive impairment, with a mean MMSE score of 17.9 points. Some of the more severely demented patients were not able to perform the Demtect and CDT as they did not understand the basic test-instructions. The specific underlying types of dementia are presented in Table 1.

\begin{tabular}{|c|c|c|c|c|c|}
\hline & n (\%) & $\mathbf{M}$ & SD & Min & Max \\
\hline \multicolumn{6}{|l|}{ Acceptance of uSense sensor } \\
\hline refused to apply sensor & $1(2.2)$ & & & & \\
\hline removed before $72 \mathrm{~h}$ by patient & $4(8.9)$ & & & & \\
\hline removed before $72 \mathrm{~h}$ by staff & $0(0)$ & & & & \\
\hline complete $72 \mathrm{~h}$ attachment & $40(88.9)$ & & & & \\
\hline \multicolumn{6}{|l|}{ Posture / Activity } \\
\hline Lying, h/day (\%) & 40 & $10.9(45)$ & $3.4(14)$ & $2.8(12)$ & $19.1(80)$ \\
\hline Sitting / standing sedentary, h/day (\%) & 40 & $9.7(41)$ & $3.1(13)$ & $1.8(7)$ & $15.4(64)$ \\
\hline Sitting / standing active, h/day (\%) & 40 & $1.7(7)$ & $0.6(3)$ & $0.3(1)$ & $2.9(12)$ \\
\hline Gait, h/day (\%) & 39 & $1.7(7)$ & $1.3(6)$ & $0.3(1)$ & $6.6(28)$ \\
\hline Total steps per day & 39 & 8829 & 7428 & 1409 & 41277 \\
\hline
\end{tabular}

According to the clinical protocol (Table 3), 44 patients (97.8\%) accepted and one patient refused the initial attachment of the uSense sensor device. Five patients removed the sensor device before the end of the three day period. Sensors were found in the patients' bathroom $(n=2)$, the social room of the ward $(n=1)$ or were handed in to the nursing staff $(n=2)$. During the 72 hours, there was no need to remove the sensor due to adverse skin reaction, pressure ulcer or intercurrent exacerbation of psychiatric symptoms, e.g. advanced persecution mania. Two out of the 44 raw data sets were incomplete or deficient, hence the technical feasibility of acquiring sensor data was $95.5 \%$. The analysis of the patients' motor behavior is presented in Table 3. Due to self-removal of the sensor, measurement days of two patients were missing. In consequence the patients' body postures were analyzed based on the remaining 40 data sets. One patient using a 4-wheeled-walker for locomotion was excluded in the analysis of gait-phases and steps per day, as this prevented an adequate stepdetection. Apart from this patient, the characteristics of patients' motor behavior are consistent with clinical 
observations reported within nursing protocols (see Table 3 and Figure 1). The following two cases further illustrate the usefulness of the sensor-based analyses:

- Male, 79 years, mixed type of dementia; MMSE: 17/30 - Nursing-protocol: 'general restlessness on ward; patient has to be brought back to the table, because he permanently rises from the chair and walks around during lunch; can't sit longer for more than one minute'. Sensor-analysis: 6.6 h/day walking; 1 h/day sitting; 41,277 steps/day

- $\quad$ Female, 74 years, Alzheimer's disease; MMSE: 15/30 - Nursing-protocol: 'from 04.45 a.m - 08.45 a.m.: disorientation and nightly psychomotor agitation; patient lays down in different beds because of disorientation; stressed behavior' Sensor-analysis: from 04.45 a.m - 08.45 a.m.: 2.6 h walking, 14,950 steps

Fig 1: Boxplot illustration of uSense-derived patients' mobility-related motor behavior [mean total hours/posture/day] ( $N=40: N=39$ for 'Gait and 'Steps' (see text for further explanation))
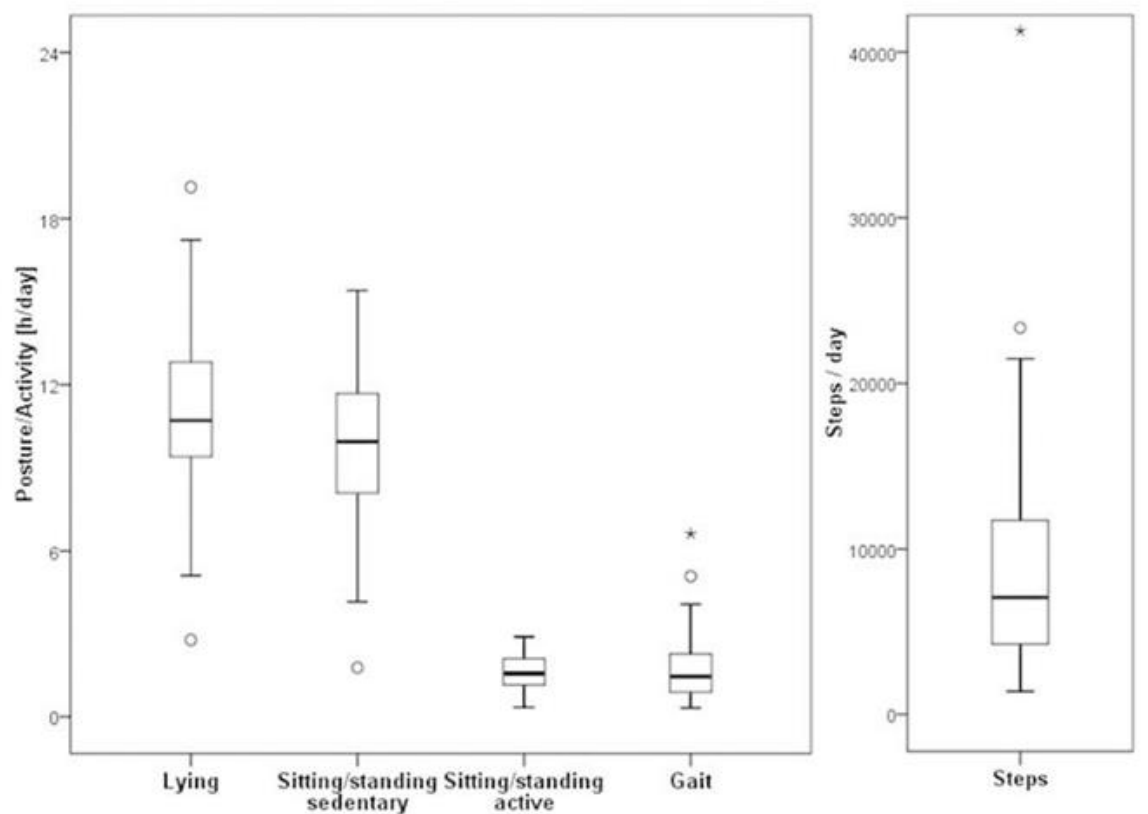

\section{DISCUSSION}

This cross-sectional study's aim was to investigate the feasibility, acceptance and relevance of hybrid motion sensors when assessing motor activity patterns in patients with of dementia during their hospital stay in an acute hospital setting.

This is the first investigation in this patient population monitoring patients' physical activity derived from body-worn sensor data based on the analyses of the sequence of specific body postures rather than actigraphy. The overall success rate in realizing the 72 hour measurements with motion sensors attached with adhesive foil on the patients' lower back was generally high (i.e. $88.9 \%$; see Table 3 ). This may be due to the aspect that the sensors attached on the patients' lower back with adhesive foil were not obtrusive and most of the patients did forget that they have attached anything on their back. The adherence to wearing the sensor and the absence of adverse skin reactions in our participants demonstrate that the attachment of the sensor device on the patients' lower back offers a unique chance to monitor motor behavior in patients suffering from dementia. In previous studies using wrist- worn actigraphs removal or total refusal have been a major problem in patients with dementia (Camargos et al., 2013). Serfaty et al. (2002) reported that more than one third of their patients suffering from dementia refused to wear the device or had them removed. We found the lower-back attached 
hybrid sensors to be more accepted than wrist-worn accelerometers that have previously been applied in dementia research.

The analysis of the sensor data reveals a wide spectrum of activity patterns (Table 3; Figure 1). Comparing the average daily sedentary time of $20.6 \mathrm{~h}(85.8 \%)$, including sedentary sitting or standing as well as lying, to 3.4 $\mathrm{h}(14.2 \%)$ of active time, including active sitting or standing and gait, indicates a very low level of physical activity in general in hospitalized patients with exacerbated BPSD. These results are comparable to a trial by Schwenk et al. (2014), who investigated patients' physical activity when discharged from a geriatric rehabilitation ward either to their home $(88.3 \%)$ or to a nursing home $(11.7 \%)$. These patients had worn an inertial sensor device, similar to our sensor-set-up, attached on the chest for 24 consecutive hours. With over $80 \%$ of sedentary time during daytime (lying + inactive sitting or standing), there is a comparable level of physical activity seen in both studies. Patients in our study spent more time during the day walking (7.2\% vs. $4.5 \%$ in Schwenk et al.) with a wider spectrum of this walking behavior respectively (SD 6\% vs 3\% in Schwenk et al.). These differences may be caused by the more advanced stage of cognitive impairment in our study sample (mean MMSE=17.9/30 compared to 22/30 in Schwenk et al.), as well as the different stage of dementia care (i.e. patients in an acute hospital setting versus community-dwelling patients).

We were surprised to see most patients with an exacerbation of BPSD lying and sitting sedentary for most time of the day. Whether this is due to psychopharmaceutical (over-) sedation, lack of therapeutical stimulation or simply caused by the hospital setting itself needs further clarification. The fact that most patients had immanent severe geriatric and other somatic problems, with sarcopenia, dehydration and gait disturbances frequently observed, might be important. Some of the patients had periods of gait instability or immobility. This might have contributed to the sedentariness seen most of our patients. However, all patients initially managed to perform the TuG-test, indicating an overall good mobility.

Some of our patients were generally restless and hyperactive, e.g. patients with more than 40,000 steps/day going straight up and down the floor or patients taking 15,000 steps on the floor within 150 minutes in the early morning hours. Clinicians and hospital staff often face such problematic motor behavior symptoms in patients with dementia. The analysis of objectively assessed motor activity patterns might, in future, help to better plan and control pharmacological as well as non-pharmacological treatment approaches. The consistency of the sensor-derived physical activity patterns and the clinical protocol indicates a good basis for the use of such body-worn sensors as an objective tool in clinical routine.

Our data acquisition and analysis protocol presently requires additional personnel in comparison to common clinical care - e.g. to initialize and start the sensor recording requires multiple steps; downloading and converting data requires two hours; and the first steps in data analysis can only be conducted with specific knowledge. However, less personnel is needed if parts of the sensor handling in clinical use (e.g. resetting the memory and starting the data acquisition) and data processing are being further automated. The detection of shuffling while leaning on a walker remains a challenge: During this type of locomotion there are only marginal vertical trunk accelerations. Hence, the accelerations measured at the lower back do allow for step detection when the patient is shuffling. In these patients the use of sensors attached to the ankle or thigh might be a more appropriate method.

As this trial reports the results of a new assessment method in hospital dementia care, it is important to state the following limitations for this investigation: Patients with different types of dementia have been included in the sample (see Table 1). Thus a heterogeneous patient sample has been included, i.e. patients with advanced motor symptoms as well as patients without motor behavior symptoms. Furthermore it is important to note that only descriptive data analyses have been conducted. We did not yet analyze the patients' motor behavior in relations to diagnoses, symptoms or clinical ratings. Besides these aspects, the length of the recording period is a key issue; due to a limited data storage and power supply, a recording phase of $72 \mathrm{~h}$ has been applied for this trial. This recording period is in accordance with Ward et al. (2005) who state a minimum period of 3 days is required to analyze patterns of motor behavior. Since there was no indication of the acceptability of our method in this population, our study took the $72 \mathrm{~h}$ as a starting point. Our study shows the feasibility of our sensor-based assessment of motor behavior in hospital dementia care over $72 \mathrm{~h}$. However, depending on 
specific goals, further analyses of the patients' mobility related motor behavior may require a longer period of data recording.

Beside these limitations, our study indicates an excellent feasibility of using lower back fixed sensor devices in clinical dementia care. The assessment of motor behavior, especially mobility, is currently a key aspect in geriatric health care and will continue to be in the future. In many countries national guidelines for mobility facilitation are being prepared or have yet to be established (The German Network for Quality Development in Nursing, 2014) focusing on mobility and motor behavior in cross-sectoral healthcare and explicitly including patients with dementia. Furthermore, the application of body-fixed sensors could provide reliable data in the assessment and objective rating of mobility-related neuropsychiatric symptoms in patients suffering from dementia (Kirste et al., 2013; Pan et al., 2013). The analysis of the patients' body postures over long time periods (days/week) offers a valuable progress in the assessment of circadian motor behavior in patients with dementia. This approach has more potential compared to the wrist-worn actigraphy (van Someren, 2009) and the commonly used geriatric assessment rating scales (King-Kallimanis et al., 2010). Along with advanced analysis methods, the use of hybrid body-fixed motion sensors may provide a basis for tailoring and evaluating innovative therapeutic approaches more effectively in hospital care with patients suffering from dementia.

\section{ACKNOWLEDGMENTS}

The authors would like to thank all participants, caregivers and legal guardians for their willingness to take part in this investigation. We also thank the board of directors of the LVR-hospital cologne and the staff of the department of geriatric psychiatry for their support in this trial.

Conflict of Interest: The authors declare no conflict of interest. Sabato Mellone owns a share in the company mHealth technologies srl, Bologna, Italy. mHealth technologies srl, among other things, develops wearable solutions for monitoring, assessment and rehabilitation of the motor function.

Author Contributions: Fleiner: study concept, data acquisition, statistical analysis, interpretation of data, draft and revision of the manuscript. Haussermann: study concept, data acquisition, clinical interpretation of data, draft and revision of the manuscript. Mellone: analysis of the sensor raw data, revision of manuscript. Zijlstra: study concept, further data analysis, interpretation of data, draft and revision of the manuscript.

Sponsor's Role: This investigation was partly funded by a young researchers funding of the German Sport University Cologne (TF), SFB 654, Teilprojekt 14 (PH), FARSEEING (TF, SM, WZ) and the institutional budgets of the authors. The funding agencies did not play any active role in the scientific investigation and reporting of the study. All authors had complete independence in this study.

\section{References}

Algase, D.L., Beattie, E.R.A., Leitsch, S.A. and Beel-Bates, C.A. (2003), "Biomechanical activity devices to index wandering behavior in dementia", American Journal of Alzheimer's Disease and Other Dementias, Vol. 18 No. 2, pp. 85-92.

Camargos, E.F., Louzada, F.M. and Nóbrega, O.T. (2013), "Wrist actigraphy for measuring sleep in intervention studies with Alzheimer's disease patients: application, usefulness, and challenges", Sleep medicine reviews, Vol. 17 No. 6, pp. 475-488.

Cohen-Mansfield, J. and Billig, N. (1986), “Agitated behaviors in the elderly. I. A conceptual review”, Journal of the American Geriatrics Society, Vol. 34 No. 10, pp. 711-721.

Cummings, J.L., Mega, M., Gray, K., Rosenberg-Thompson, S., Carusi, D.A. and Gornbein, J. (1994), “The Neuropsychiatric Inventory: comprehensive assessment of psychopathology in dementia", Neurology, Vol. 44 No. 12 , pp. 2308-2314. 
The German Network for Quality Development in Nursing (DNQP) (2014), "Experts Standard preservation and promotion of mobility in nursing: working documents for the conference to Expertenstandard after 113a SGB XI", available at: http://www.wiso.hsosnabrueck.de/fileadmin/groups/607/Arbeitsunterlagen_Fachkonferenz.pdf (accessed 17 April 2014).

Draper, B., Finkel, S.I. and Tune, L. (2015), “An introduction to BPSD”, in The IPA Complete Guides to Behavioral and Psychological Symptoms of Dementia: Specialists Guide, Module I, pp. 1.1-1.13.

Eriksson, S., Strandberg, S., Gustafson, Y. and Lundin-Olsson, L. (2009), "Circumstances surrounding falls in patients with dementia in a psychogeriatric ward", Archives of Gerontology and Geriatrics, Vol. 49 No. 1, pp.80-87.

FARSEEING EU-Consortium (2015), "FAll Repository for the design of Smart and sElf-adaptive Environments prolonging INdependent livinG. European Commission funded research project with 10 partners distributed in 5 EU countries", available at: http://farseeingresearch.eu/ (accessed 9 July 2015).

Fleiner, T., Zijlstra, W., Dauth, H. and Haussermann, P. (2015), "Evaluation of a hospital-based daystructuring exercise programme on exacerbated behavioural and psychological symptoms in dementia - the exercise carrousel: study protocol for a randomised controlled trial”, Trials, Vol. 16 No. 1, p. 228.

Folstein, M.F., Folstein, S.E. and McHugh, P.R. (1975), “"Mini-mental state”. A practical method for grading the cognitive state of patients for the clinician", Journal of Psychiatric Research, Vol. 12 No. 3, pp. 189-198.

Halek, M. and Bartholomeyczik, S. (2012), "Description of the behaviour of wandering in people with dementia living in nursing homes - a review of the literature", Scandinavian journal of caring sciences, Vol. 26 No. 2, pp. 404-413.

Hindmarch, I., Lehfeld, H., Jongh, P. de and Erzigkeit, H. (1998), "The Bayer Activities of Daily Living Scale (B-ADL)", Dementia and Geriatric Cognitive Disorders, 9 Suppl 2, pp. 20-26.

Inouye, S.K., van Dyck, C H, Alessi, C.A., Balkin, S., Siegal, A.P. and Horwitz, R.I. (1990), "Clarifying confusion: the confusion assessment method. A new method for detection of delirium", Annals of Internal Medicine, Vol. 113 No. 12, pp. 941-948.

Kalbe, E., Kessler, J., Calabrese, P., Smith, R., Passmore, A.P., Brand, M. and Bullock, R. (2004), “DemTect: a new, sensitive cognitive screening test to support the diagnosis of mild cognitive impairment and early dementia”, International Journal of Geriatric Psychiatry, Vol. 19 No. 2, pp. 136-143.

Kales, H.C., Gitlin, L.N. and Lyketsos, C.G. (2015), "Assessment and management of behavioral and psychological symptoms of dementia", BMJ (Clinical research ed.), Vol. 350, pp. h369.

King-Kallimanis, B., Schonfeld, L., Molinari, V.A., Algase, D., Brown, L.M., Kearns, W.D., Davis, D.M., Werner, D.H., Beattie, E.R. and Nelson, A.L. (2010), "Longitudinal investigation of wandering behavior in Department of Veterans Affairs nursing home care units", International Journal of Geriatric Psychiatry, Vol. 25 No. 2, pp.166-174.

Kirste, T., Hoffmeyer, A., Koldrack, P., Bauer, A., Schubert, S., Schröder, S. and Teipel, S. (2013), "Detecting the Effect of Alzheimer's Disease on Everyday Motion Behavior", Journal of Alzheimer's disease, pp. 121132.

Lyketsos, C.G., Carrillo, M.C., Ryan, J.M., Khachaturian, A.S., Trzepacz, P., Amatniek, J., Cedarbaum, J., Brashear, R. and Miller, D.S. (2011), "Neuropsychiatric symptoms in Alzheimer's disease", Alzheimer's \& Dementia The Journal of the Alzheimer's Association, Vol. 7 No. 5, pp. 532-539.

Morgenthaler, T., Alessi, C., Friedman, L., Owens, J., Kapur, V., Boehlecke, B., Brown, T., Chesson, A.,Coleman, J., Lee-Chiong, T., Pancer, J. and Swick, T.J. (2007), "Practice parameters for the use of actigraphy in the assessment of sleep and sleep disorders: an update for 2007", Sleep, Vol. 30 No. 4, pp. 519529. 
Pan, W.-D., Yoshida, S., Liu, Q., Wu, C.-L., Wang, J., Zhu, J. and Cai, D.-F. (2013), "Quantitative evaluation of severity of behavioral and psychological symptoms of dementia in patients with vascular dementia", Translational neurodegeneration, Vol. 2 No. 1, p. 9.

Podsiadlo, D. and Richardson, S. (1991), "The timed "Up \& Go": a test of basic functional mobility for frail elderly persons", Journal of the American Geriatrics Society, Vol. 39 No. 2, pp. 142-148.

Ries, J.D., Echternach, L.N. and Gagon Blodgett, M. (2009), "Test-Retest Reliability and Minimal Detectable Change Scores for the Timed "Up \& Go" Test, the Six-Minute Walk Test, and Gait Speed in People With Alzheimer Disease", Physical Therapy, Vol. 89 No. 6, pp. 569-579.

Sasaki, J.E., John, D. and Freedson, P.S. (2011), "Validation and comparison of ActiGraph activity monitors", Journal of science and medicine in sport / Sports Medicine Australia, Vol. 14 No. 5, pp. 411-416.

Schwenk, M., Hauer, K., Zieschang, T., Englert, S., Mohler, J. and Najafi, B. (2014), "Sensor-derived physical activity parameters can predict future falls in people with dementia", Gerontology, Vol. 60 No. 6, pp. 483492.

Serfaty, M., Kennell-Webb, S., Warner, J., Blizard, R. and Raven, P. (2002), "Double blind randomised placebo controlled trial of low dose melatonin for sleep disorders in dementia", International journal of geriatric psychiatry, Vol. 17 No. 12, pp. 1120-1127.

Stern, Y. (2002), "What is cognitive reserve? Theory and research application of the reserve concept", Journal of the International Neuropsychological Society, Vol. 8 No. 3, pp. 448-460.

Sunderland, T., Hill, J.L., Mellow, A.M., Lawlor, B.A., Gundersheimer, J., Newhouse, P.A. and Grafman, J.H. (1989), "Clock drawing in Alzheimer's disease. A novel measure of dementia severity", Journal of the American Geriatrics Society, Vol. 37 No. 8, pp. 725-729.

van Someren, E.J.W. (2009), "Ambulatory monitoring of human behavior, physiology and environment - A Research and Development agenda to go beyond actigraphy", in Magjarevic, R., Dössel, O. and Schlegel,W.C. (Eds.), World Congress on Medical Physics and Biomedical Engineering, September 7 - 12, 2009, Munich, Germany, IFMBE Proceedings, 25/7, Springer Berlin Heidelberg, Berlin, Heidelberg, pp. 362-365.

Van Someren, E. J. W. (2011), “Actigraphic monitoring of sleep and circadian rhythms”, Handbook of clinical neurology, Vol. 98, pp. 55-63.

Ward, D.S., Evenson, K.R., Vaughn, A., Rodgers, A.B. and Troiano, R.P. (2005), “Accelerometer Use in Physical Activity: Best Practices and Research Recommendations", Medicine \& Science in Sports \& Exercise, Vol. 37 No. Supplement, pp. S582-S588.

World Health Organisation (WHO) (1997), The ICD-10 classification of mental and behavioural disorders: ICD-10;clinical descriptions and diagnostic guidelines, Reprinted, Geneva.

Zijlstra, W. and Aminian, K. (2007), "Mobility assessment in older people: new possibilities and challenges", European Journal of Ageing, Vol. 4 No. 1, pp. 3-12.

Zijlstra, W., Becker, C. and Pfeiffer, K. (2011), "Wearable systems for monitoring mobility related activities; from technology to application for healthcare services", in Ziefle, M. (Ed.), E-health, Assistive Technologies and Applications for Assisted Living: Challenges and Solutions, IGI Global, Hershey, Pennsylvania, pp. 245268.

Zuidema, S.U., Derksen, E., Verhey, F.R. and Koopmans, R.T. (2007), "Prevalence of neuropsychiatric symptoms in a large sample of Dutch nursing home patients with dementia", International Journal of Geriatric Psychiatry, Vol. 22 No. 7, pp. 632-638. 